\title{
RESISTÊNCIA DE SOJA A INSETOS: VI. COMPORTAMENTO DE GENÓTIPOS EM RELAÇÃo A PERCEVEJOS ( ${ }^{1}$ )
}

\author{
CARLOS JORGE ROSSETTO $(2,5)$, TOSHIO IGUE $\left({ }^{3}\right)$, \\ MANOEL ALBINO COELHO DE MIRANDA $(4,5)$ e ANDRÉLUIZLOURENÇĀO $(2,5)$
}

\begin{abstract}
RESUMO
O comportamento de trinta e cinco genótipos de soja foi estudado em condiçōes de campo, em Campinas, SP, em relação ao ataque dos percevejos pentatomideos Euschistus heros (F.), Piezodorus guildinii (Westwood) e Nezara viridula (L.). A infestação natural do campo experimental foi suplementada com a liberação de percevejos adultos. Em 1983/1984, o dano médio sofrido pelos genótipos foi moderado e, em 1984/1985, severo. Três critérios foram utilizados para avaliar a resistência das variedades: a porcentagem de área foliar retida após a maturação das vagens, o índice porcentual de dano de vagem e a produção de grāos. A testemunha suscetível usada foi o cultivar Paraná, que produziu apenas $402 \mathrm{~kg} / \mathrm{ha}$ e $31 \mathrm{~kg} / \mathrm{ha} \mathrm{em} 1983 / 1984$ e 1984/1985 respectivamente. A linhagem IAC 80/4228 apresentou uma resistência estável, tendo produzido $1.675 \mathrm{~kg} / \mathrm{ha}$ e $1.639 \mathrm{~kg} / \mathrm{ha}$ em $1983 / 1984$ e $1984 / 1985$ respectivamente.
\end{abstract}

Termos de indexação: soja, Glycine max (L.) Merrill, percevejos, Pentatomidae, resistência de planta, Piezodorus guildinï (Westwood), Nezara viridula (L.), Euschistus heros $(\mathrm{F}$.$) .$

(1) Trabalho apresentado no X Congresso Brasileiro de Entomologia, 26-31 de janeiro de 1986, Rio de Janeiro. Recebido para publicação em 11 de novembro de 1985.

(2) Seção de Entomologia Fitotécnica, Instituto Agronómico (IAC), Caixa Postal 28, 13001 Campinas (SP), Brasil.

(3) Seção de TÉcnica Experimental e Cálculo, LAC.

(4) Seção de Leguminosas, LAC.

(5) Com bolsa de suplementação do CNPq. 


\section{INTRODUÇÃO}

O controle de insetos através de genótipos resistentes é ideal pelas seguintes razões (ROSSETTO et alii, 1981): (1) Ė gratuito para o agricultor, representando custo apenas para o Governo ou para os produtores de semente que realizam a pesquisa. Por esta razão, o controle de pragas com variedades resistentes representa uma economia para o agricultor e uma possibilidade de aumento de sua renda líquida. (2) Não é poluente nem acarreta problemas de resíduos tóxicos nos alimentos produzidos. (3) Não apresenta problemas de transferência de tecnologia, indo através da semente melhorada diretamente da pesquisa para o usuário. (4) Beneficia indistintamente todos os agricultores, pequenos ou grandes, pois não é uma medida de controle que requeira investimento de infra-estrutura ou máo-de-obra para ser aplicada por parte do produtor. (5) Atua contra qualquer nivel de infestação da praga, nāo havendo necessidade de aguardar a população atingir o nivel de dano econômico para ser utilizado. (6) Não interfere com outras práticas agricolas, especialmente com a colheita, inexistindo tempo de carência entre sua aplicação e a data da colheita. (7) Em geral é uma tecnologia nacional. (8) O uso de cultivar resistente, por via de regra, é compativel com qualquer outra medida de controle que necessite ser usada na lavoura.

Por essas razões, o cultivar resistente é um componente desejável em todo programa racional e cientificamente elaborado de controle de pragas, atualmente chamado de manejo integrado, controle integrado ou simplesmente manejo.

Foi iniciado, em 1976, no Instituto Agronômico, um programa de pesquisas com participação das Seçōes de Entomologia Fitotécnica e Leguminosas, com o objetivo de obter cultivares de soja resistentes a insetos, especialmente a percevejos pentatomídeos, que constituem a principal praga da cultura no Estado de São Paulo. Nesse programa de melhoramento, têm sido utilizadas como fontes de resistência linhagens derivadas do cruzamento Hill x PI 274454, sobretudo as linhagens IAC73-227, IAC73-228 e IAC73-231 (MIRANDA et alii, 1979; PANIZZI et alii, 1981; ROSSETTO et alii, 1984; LOURENÇĀO et alii, 1985).

O objetivo deste trabalho foi avaliar o comportamento em relação a percevejos dos principais cultivares de soja plantados no Estado de São Paulo, assim como de algumas introduçóes e de linhagens já obtidas pelo referido programa de melhoramento.

\section{MATERIAL E MÉTODOS}

Foram feitos dois experimentos em condições de campo no Centro Experimental de Campinas, o primeiro plantado em 18 de novembro de 1983 e o segundo, em 22 de novembro de 1984. 
Utilizaram-se treze linhagens selecionadas para resistência a percevejos (IAC80/55-1, IAC80/95-0-2, IAC80/596-2, IAC80/612-5-2, IAC80/598-2-4, IAC80/ 1508-3, IAC80/572-2-1, IAC78/2318, IAC78/2286, IAC79/1823, IAC80/4228, IAC80/ 14213 e IAC73/228), dezesseis cultivares (Paraná, UFV-1, IAC-2, IAC-7, IAC-8, IAC-9, IAC-10, IAC-11, IAC-12, IAC Santa Maria 702, IAC Foscarim-31, Santa Rosa, Cristalina, IAS-5, Bossier e São Carlos) e seis introduções (PI 171451, PI 227687, PI 229358, PI 274453, PI 274454 e D72/9601-1).

Utilizou-se delineamento em blocos completos ao acaso com 35 tratamentos e quatro repetiçōes. A parcela foi constituida de uma linha de $2 \mathrm{~m}$ de comprimento. Plantaram-se 50 sementes por metro, inoculadas com Rhizobium japonicum. No desbaste, deixaram-se vinte plantas por metro. Fez-se adubação no sulco de plantio, utilizando-se uma fórmula granulada com 0-28-15 de NPK e aplicando-se 12,5 gramas por metro linear.

Para evitar acamamento, os tratamentos mais altos foram mantidos eretos mediante armação de tutores de bambu.

Quando os cultivares mais precoces iniciaram a formação de vagens, foram liberados trinta adultos de percevejos pentatomídeos por parcela, ou seja, cinco adultos por metro linear. No segundo experimento, além dessa liberação, foi feita uma segunda, vinte dias depois, usando-se a mesma quantidade de insetos. No primeiro experimento (1983/1984), $75 \%$ dos percevejos liberados eram $P$. guildinii coletados em soja no Centro Experimental de Campinas, e $25 \%, N$. viridula criados sobre soja. No segundo experimento (1984/1985), $80 \%$ dos percevejos liberados eram $E$. heros coletados em Aguaí sobre soja. Em ambos, devido à infestação natural, havia $P$. guildinii, E. heros e N. viridula no campo experimental: no primeiro, houve predominância de $P$. guildinii e no segundo, de $E$. heros. Não se fez nenhuma pulverização de inseticida ou fungicida nos dois ensaios.

Utilizaram-se três critérios para comparar a resistência dos tratamentos: o índice porcentual de dano de vagens (!PDV); a porcentagem de retenção foliar (PRF) e o peso de grãos comerciais.

IPDV - Após a maturação das vagens, retirou-se uma amostra de cem vagens de cada parcela, da região média das plantas (ROSSETTO et alii, 1984), procurando-se retirar vagens de vinte ou mais plantas da linha central da parcela, para determinar o índice porcentual de dano de vagens (IPDV) através da fórmula:

IPDV $=1 / 2$ (\% vagens intermediárias $)+\%$ vagens planas.

Em trabalho anterior (ROSSETTO et alii, 1984), o indice de dano de vagens (IDV) foi obtido pela fórmula:

IDV $=\%$ vagens intermediárias +2 (\% vagens planas).

O IDV assim definido varia de 0 a 200 . Dividindo-o por 2 , ele vai variar de 0 a 100. Esse índice, que se denominou indice porcentual de dano de vagens (IPDV $=$ IDV/2), foi utilizado neste trabalho para comparar o dano causado às 
vagens pelos percevejos. O IPDV é mais racional, pois equivale a se atribuir peso zero a vagens sadias, peso um a vagens totalmente danificadas e peso meio a vagens intermediárias.

PRF - No mesmo dia da retirada da amostra de vagens, atribuiu-se visualmente a cada parcela uma porcentagem de retenção foliar (PRF).

Peso - Colocaram-se as plantas colhidas dentro de um saco de tela plástica, malhando-as com um bastão de madeira. A seguir, peneiraram-se e escolheram-se os grãos, eliminando-se os tipos 3 e 4, deformados pela ação dos percevejos, e pesaram-se os grãos comerciais, tipo 1 e 2 (ROSSETTO et alii, 1984).

Para fins de análise estatistica, o IPDV e a PRF foram transformados em arco seno $\sqrt{(\text { IPDV ou PRF)/100. }}$

\section{RESULTADOS E DISCUSSÃO}

Os tratamentos estudados, suas origens, altura e ciclo do plantio até a colheita estão no quadro 1. O ciclo em geral foi mais longo devido ao ataque de percevejos. O do cultivar Paraná, por exemplo, quando não infestado por percevejos, é de 120 dias; com infestação, atingiu 158 dias em 1983/1984 e 144 em 1984/1985. O ponto de colheita em soja é evidenciado pela queda das folhas e maturação das vagens. A infestação de percevejos nos tratamentos suscetiveis, como o 'Paraná, provoca retenção foliar, tornando-se difícil determinar o ponto de colheita. Devido à destruição das vagens, a planta floresce novamente e produz novas vagens. Numa mesma planta atacada, encontram-se vagens já maduras e vagens ainda verdes da segunda florada, e isso também dificulta a determinação do ponto de colheita. Por essas razões, o ciclo dos genótipos neste trabatho não serve como referência nem foi analisado estatisticamente.

O quadro 2 - resultados da análise conjunta dos dois anos de experimento - mostra que o efeito de ano deu os maiores valores de $\mathrm{F}$.

No quadro 3, encontra-se a porcentagem de área foliar retida após a maturação das vagens; no quadro 4,0 indice porcentual de dano de vagens (IPDV) e, no quadro 5, a produção expressa em quilogramas de grãos comerciais por hectare.

Observa-se que o dano dos percevejos foi muito maior no segundo experimento (1984/1985) do que no primeiro. A porcentagem média de retenção foliar foi de 10,6 em 1983/1984 e de 57,5 em 1984/1985. O índice porcentual de dano de vagem (IPDV) foi de $29,4 \mathrm{em} 1983 / 1984$ e de $64,6 \mathrm{em} 1984 / 1985$. A produção média foi de $2.036 \mathrm{~kg} / \mathrm{ha}$ em 1983/1984 e de $1.010 \mathrm{~kg} / \mathrm{ha}$ em 1984/1985. 
QUADRO 1. Origem, ciclo médio do plantio à colheita e altura média do germoplasma de soja. Campinas

\begin{tabular}{|c|c|c|c|c|c|}
\hline \multirow{2}{*}{ Tratamento } & \multirow{2}{*}{ Origem } & \multicolumn{2}{|c|}{ Ciclo } & \multicolumn{2}{|c|}{ Altura } \\
\hline & & $1983 / 1984$ & $1984 / 1985$ & $1983 / 1984$ & $1984 / 1985$ \\
\hline IAC 80-55-1 & Paraná x IAC 73-228* & 141 & 146 & 65 & 64 \\
\hline IAC 80-95-0-2 & Paraná x IAC 73-228* & 141 & 146 & 74 & 70 \\
\hline IAC $80-596-2$ & IAC $73-228^{*} \times$ UFV -1 & 141 & 162 & 59 & 56 \\
\hline IAC $80-612-5-2$ & IAC 73-228* $x$ UFV -1 & 158 & 162 & 60 & 61 \\
\hline IAC 80-598-2-4 & IAC 73-228*x UFV-1 & 158 & 156 & 60 & 60 \\
\hline IAC 80-508-3 & Paraná x IAC 73-228* & 141 & 144 & 60 & 65 \\
\hline IAC 80-572-2-1 & IAC $7 \times$ IAC 73-228* & 146 & 154 & 62 & 69 \\
\hline IAC 78-2318 & D72-9601 x IAC 73-227* & 146 & 151 & 61 & 65 \\
\hline IAC $78-2286$ & D72-9601 x UFV.-1 & 158 & 166 & 62 & 67 \\
\hline Paraná & Hill x (Roanoke x Ogden) & 158 & 144 & 66 & 60 \\
\hline UFV-I & Seleção em Viçoja & 158 & 167 & 70 & 80 \\
\hline IAC-2 & La 41-1219 x Yelnando & 158 & 167 & 116 & 122 \\
\hline IAC-7 & Seleçāo em RB 72-1** & 158 & 165 & 85 & 94 \\
\hline IAC-8 & Bragg $\times$ (Hill $\times$ PI 240664) & 159 & 165 & 81 & 90 \\
\hline IAC-9 & Seleção em RB 72-1** & 168 & 167 & 101 & 101 \\
\hline IAC -10 & Hardee $x$ Hill & 158 & 164 & 71 & 76 \\
\hline IAC-11 & Parana $\times$ (Davis $\times$ IAC 73-1334)*** & 158 & 162 & 97 & 99 \\
\hline IAC-12 & Parana $x$ IAC 73-231* & 158 & 146 & 69 & 70 \\
\hline Santa Rosa & D49-772 x La 41-1219 & 158 & 163 & 79 & 72 \\
\hline Cristalina & Provavel UFV -1 × Davis & 158 & 166 & 94 & 97 \\
\hline IAC Foscarim-31 & Seleçāo em Halle-7 & 158 & 146 & 85 & 87 \\
\hline IAS-5 & Hill x (Roanoke x Ogden) & 148 & 144 & 55 & 54 \\
\hline Bossier & Seleçáo em Lee & 154 & 149 & 59 & 61 \\
\hline IAC Santa Maria 702 & Seleçāo em Santa Maria & 155 & 167 & 117 & 120 \\
\hline IAC 79-1823 & IAC 70-308 x D72-9601 & 158 & 168 & 82 & 99 \\
\hline IAC $80-4228$ & IAC 73-231* x Santa Rosa & 161 & 163 & 101 & 102 \\
\hline IAC 80.4213 & IAC 73-231* x Santa Rosa & 158 & 157 & 65 & 69 \\
\hline PI 171451 & Japม̃o & 141 & 145 & 47 & 31 \\
\hline PI 227687 & Okinawa, Japão & 140 & 139 & 150 & 145 \\
\hline PI 229358 & Tóquio, Japão & 141 & 144 & 47 & 26 \\
\hline PI 274454 & Okinawa, Japäo & 186 & 170 & 185 & 170 \\
\hline PI 274453 & Okinawa, Japåo & 186 & 180 & 172 & 167 \\
\hline IAC 73-228 & Hill x PI 274454 & 158 & 154 & 66 & 62 \\
\hline D72-9601-1 & D66-8666 x (Bragg x PI 229358) & 158 & 151 & 51 & 45 \\
\hline Sāo Carlos & Seleção em Davis & 158 & 154 & 69 & 66 \\
\hline Média & & 155,2 & 157,1 & 81,4 & 81,3 \\
\hline
\end{tabular}

- IAC 73-227, IAC 73-228 e IAC 73-231 foram originadas de Hill x PI 274454.

* RB significa Romeu Bulk. Este bulk foi composto a partir dos seguintes cruzamentos: E70-46 x Viçoja; E70-46 x Pickett, E70-47 $x$ Viçoja, E70-47 x F65-1376, Hill x E70-47, Davis x E70-48. As linhagens E70-46, E70-47, E70-48 foram selecionadas do cruzamento Hill x PI 240664.

** IAC 73-1334 foi originada de Davis x (Hill x PI 240664).

A infestação de percevejos em 1983/1984 foi moderada, enquanto em 1984/1985 foi forte. Isso se refletiu em sintomas de dano maiores e produção menor no segundo ano do experimento. Houve efeito significativo dos genótipos (Quadro 2), confirmando a expectativa de que alguns materiais são mais resistentes a percevejos que outros. O maior valor do teste F para genótipos foi do IPDV (Quadro 2), mostrando que este índice é um critério bom e prático para discriminar variedades resistentes e suscetiveis. A interação genótipo $x$ ano foi significativa (Quadro 2), 
talvez por ter o nivel de infestação sido moderado no primeiro ano e severo no segundo e por ter predominado no primeiro ano a espécie $P$. guildinii e, no segundo, a $E$. heros.

O quadro 6 apresenta um sumário das médias de dois anos da PRF, IPDV e produção, sem análise estatística; todavia, como ocorreu interação signiificativa com ano, a discriminação estatística das médias foi feita dentro de cada ano.

A infestação severa do segundo ano do experimento permitiu melhor discriminação dos tratamentos, sendo as médias ordenadas com base nele. O cultivar Paraná confirmou sua alta suscetibilidade a percevejos, sendo recomendado para uso como testemunha suscetivel. A linhagem IAC73-228, utilizada como fonte de resistência a percevejos, confirmou sua resistência. Duas linhagens obtidas no programa de melhoramento contra percevejos se destacaram: a IAC80-4228, derivada do cruzamento IAC73-231 x Santa Rosa, e a IAC 80-598-2-4, derivada do cruzamento IAC73-228 x UFV-1 (LOURENÇĀO et alii, 1985). A IAC80-4228 mostrou boa estabilidade na produção (Quadro 5): ela apresenta defeito grave de acamamento, não avaliado no presente estudo porque se utilizaram tutores para escorar os genótipos mais altos. A linhagem IAC80-598-2-4 é baixa (Quadro 1) e sensivel a fotoperiodo. Por causa desses defeitos, não podem ser recomendadas para cultivo, conquanto apresentem boas caracteristicas para emprego em programa de melhoramento.

QUADRO 2. Valores do coeficiente de variação da média geral e do teste F obtido da análise conjunta de dois experimentos, realizados em 1983/1984 e 1984/1985, com 35 genótipos de soja infestados por percevejos. Campinas

\begin{tabular}{|c|c|c|c|}
\hline \multirow[b]{2}{*}{ Fatores } & \multicolumn{3}{|c|}{ Variáveis } \\
\hline & $\begin{array}{c}\text { Índice } \\
\text { porcentual } \\
\text { de dano } \\
\text { de vagem }\end{array}$ & $\begin{array}{l}\text { Porcentagem } \\
\text { de retenção } \\
\text { foliar }\end{array}$ & Produção \\
\hline Ano . . . . . . . . . . . & $59,8 *$ & $65,5^{*}$ & $39,1^{*}$ \\
\hline Genótipos . . . . . . . . & $10,1^{*}$ & $3,3^{*}$ & $4,9^{*}$ \\
\hline Ano $x$ Genotipo ........ & $4,4^{*}$ & $5,3^{*}$ & $3,2^{*}$ \\
\hline Coeficiente de variação .... & $12,5 \%$ & $38,1 \%$ & $29,5 \%$ \\
\hline Média geral . . . . . . . . & $43,7 \%$ & $33,0 \%$ & $1523 \mathrm{~kg} / \mathrm{ha}$ \\
\hline
\end{tabular}

* Valores do teste F significativos ao nivel de $5 \%$. 
QUADRO 3. Porcentagem média de retenção foliar (PRF) em 35 germoplasmas de soja infestados por percevejos (médias de quatro repetições). Campinas

\begin{tabular}{|c|c|c|}
\hline \multirow{2}{*}{ Tratamento } & \multicolumn{2}{|c|}{ Retenção foliar* } \\
\hline & $1983 / 1984$ & $1984 / 1985$ \\
\hline & $\%$ & $\%$ \\
\hline IAC $73-228 \ldots \ldots \ldots \ldots \ldots$ & $0,7 \mathbf{h}$ & $8,7 \mathrm{j}$ \\
\hline IAC $80-4228 \ldots \ldots \ldots \ldots \ldots$ & $4,2 \mathrm{gh}$ & $10,0 \mathrm{j}$ \\
\hline IAC $80-598-2-4 \ldots \ldots \ldots \ldots$ & $0,0 \mathrm{~h}$ & $10,5 j$ \\
\hline PI $274453 \ldots \ldots \ldots \ldots \ldots \ldots$ & $23,7 \mathrm{~cd}$ & $13,7 \mathrm{ij}$ \\
\hline IAC $80-508-3 \ldots \ldots \ldots \ldots$ & $0,0 \mathrm{~h}$ & $20,0 \mathrm{ij}$ \\
\hline IAC $78-2318 \ldots \ldots \ldots \ldots \ldots$ & $0,0 \mathrm{~h}$ & $22,0 \mathrm{ij}$ \\
\hline IAC $80-95-0-2 \ldots \ldots \ldots \ldots$ & $2,0 \mathrm{~h}$ & $23,2 \mathrm{ij}$ \\
\hline PI $274454 \ldots \ldots \ldots \ldots \ldots \ldots$ & $2,5 \mathrm{~h}$ & $22,5 \mathrm{hij}$ \\
\hline IAC $80-596-2 \ldots \ldots \ldots \ldots$ & $0,0 \mathrm{~h}$ & $21,2 \mathrm{hij}$ \\
\hline IAC $80-55-1 \ldots \ldots \ldots \ldots \ldots$ & $0,0 \mathrm{~h}$ & $30,2 \mathrm{ghij}$ \\
\hline IAC $-11 \ldots \ldots \ldots \ldots \ldots$ & $2,7 \mathrm{~h}$ & 27,5 ghij \\
\hline PI $171451 \ldots \ldots \ldots \ldots \ldots \ldots \ldots$ & $0,0 \mathrm{~h}$ & 28,7 ghij \\
\hline PI $227687 \ldots \ldots \ldots \ldots \ldots$ & $0,0 \mathrm{~h}$ & 30,0ghij \\
\hline IAC $80-612-5-2 \ldots \ldots \ldots \ldots \ldots$ & $0,0 \mathrm{~h}$ & $31,2 \mathrm{ghij}$ \\
\hline PI $229358 \ldots \ldots \ldots \ldots \ldots \ldots \ldots$ & $1,2 \mathrm{~h}$ & $46,2 \mathrm{fghij}$ \\
\hline IAC $78-2286 \ldots \ldots \ldots \ldots \ldots$ & $0,0 \mathrm{~h}$ & $53,7 \mathrm{efghi}$ \\
\hline IAC $80-572-2-1 \ldots \ldots \ldots \ldots \ldots$ & $0,7 \mathrm{~h}$ & $63,7 \mathrm{defgh}$ \\
\hline $\mathrm{IAC}-7 \ldots \ldots \ldots \ldots \ldots$ & $1,2 \mathrm{~h}$ & $63,7 \mathrm{cdefgh}$ \\
\hline IAC $80-4213 \ldots \ldots \ldots \ldots \ldots$ & $0,5 h$ & 62,5 bcdefg \\
\hline $\mathrm{IAC}-2 \ldots \ldots \ldots \ldots \ldots \ldots$ & $4,7 \mathrm{gh}$ & $60,0 \mathrm{bcdefg}$ \\
\hline IAC-79-1823 $\ldots \ldots \ldots \ldots \ldots$ & 6,7 efgh & $70,0 a b c d e f$ \\
\hline IAC $-10 \ldots \ldots \ldots \ldots \ldots$ & $2,5 \mathrm{~h}$ & 72,5 abcdef \\
\hline 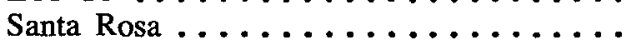 & $1,2 \mathrm{~h}$ & 80,0 abcde \\
\hline Cristalina $\ldots \ldots \ldots \ldots \ldots \ldots$ & $18,0 \mathrm{cde}$ & $86,2 \mathrm{abcde}$ \\
\hline UFV $-1 \ldots \ldots \ldots \ldots \ldots \ldots$ & $3,0 \mathrm{gh}$ & 87,5 abcde \\
\hline D72-9601-1 $\ldots \ldots \ldots \ldots \ldots \ldots$ & 7,2 fgh & $91,2 \mathrm{abcd}$ \\
\hline IAC-9 $\ldots \ldots \ldots \ldots \ldots \ldots$ & $16,2 \mathrm{cdef}$ & $92,5 \mathrm{abcd}$ \\
\hline IAC Santa Maria $702 \ldots \ldots \ldots \ldots$ & $29,5 c$ & $92,5 \mathrm{abcd}$ \\
\hline IAC $-12 \ldots \ldots \ldots \ldots$ & $22,7 \mathrm{cde}$ & $95,0 a b c$ \\
\hline IAC $-8 \ldots \ldots \ldots \ldots \ldots \ldots$ & $0,0 \mathrm{~h}$ & $96,2 \mathrm{ab}$ \\
\hline São Carlos . . . . . . . . . . . & 15,0 defg & $100,0 \mathrm{a}$ \\
\hline 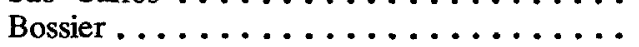 & $22,2 \mathrm{~cd}$ & $100,0 \mathrm{a}$ \\
\hline IAS $-5 \ldots \ldots \ldots \ldots \ldots \ldots \ldots$ & $69,0 b$ & $100,0 \mathrm{a}$ \\
\hline IAC Foscarim $31 \ldots \ldots \ldots \ldots$ & $22,5 \mathrm{~cd}$ & $100,0 \mathrm{a}$ \\
\hline 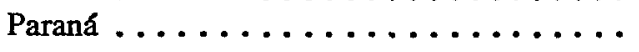 & $91,7 \mathrm{a}$ & $100,0 \mathrm{a}$ \\
\hline Média $\ldots \ldots \ldots \ldots \ldots \ldots \ldots$ & 10,6 & 57,5 \\
\hline Coeficiente de variação $\ldots \ldots \ldots \ldots \ldots$ & $51,2 \%$ & $31,3 \%$ \\
\hline
\end{tabular}

* Médias seguidas pela mesma letra não diferem entre si pelo teste de Duncan a 5\%. 
QUADRO 4. Índice porcentual de dano de vagem (IPDV) de 35 germoplasmas de soja infestados por percevejos (média de quatro repetições). Campinas

Tratamento

Índice porcentual de dano de vagem*

$1983 / 1984$

\begin{tabular}{|c|c|c|}
\hline & $\%$ & $\%$ \\
\hline PI $274453 \ldots \ldots \ldots \ldots \ldots \ldots \ldots$ & $11,6 \mathrm{pq}$ & 10,60 \\
\hline PI $274454 \ldots \ldots \ldots \ldots \ldots \ldots \ldots$ & $7,3 \mathrm{q}$ & 16,90 \\
\hline IAC $80-4228 \ldots \ldots \ldots \ldots \ldots \ldots$ & 19,9jklmnop & $32,8 \mathrm{n}$ \\
\hline IAC $80-598-2-4 \ldots \ldots \ldots \ldots$ & 16,9klmnop & $37,1 \mathrm{mn}$ \\
\hline PI $227687 \ldots \ldots \ldots \ldots \ldots \ldots \ldots$ & 12,9 nopq & $39,3 \mathrm{mn}$ \\
\hline IAC $73-228 \ldots \ldots \ldots \ldots \ldots$ & $17,9 \mathrm{klmnop}$ & $43,31 \mathrm{mn}$ \\
\hline IAC $80-612-5-2 \ldots \ldots \ldots \ldots \ldots$ & $19,4 j$ klmnop & $50,1 \mathrm{klm}$ \\
\hline IAC $78-2318 \ldots \ldots \ldots \ldots \ldots$ & $14,5 \mathrm{mnopq}$ & $50,8 \mathrm{klm}$ \\
\hline IAC $80-4213 \ldots \ldots \ldots \ldots$ & $12,3 \mathrm{opq}$ & $51,0 \mathrm{klm}$ \\
\hline IAC $80-596-2 \ldots \ldots \ldots \ldots$ & 16,4lmnop & $53,0 \mathrm{jklm}$ \\
\hline Santa Rosa . . . . . . . . . . . . . & $21,5 i j k l m n o$ & $57,1 \mathrm{ijkl}$ \\
\hline IAC $-7 \ldots \ldots \ldots \ldots \ldots \ldots$ & 22,0 ijklmn & $58,6 \mathrm{hijkl}$ \\
\hline IAC $80-572-2-1 \ldots \ldots \ldots \ldots \ldots$ & 19,5jklmnop & 58,9 hijkl \\
\hline IAC $80-95-0-2 \ldots \ldots \ldots \ldots \ldots$ & 26,3ghijkl & $59,6 \mathrm{ghijkl}$ \\
\hline IAC-9 . . . . . . . . . . . & 27,0 ghijk & $60,9 \mathrm{ghijkl}$ \\
\hline IAC $-80-55-1 \ldots \ldots \ldots \ldots \ldots$ & $22,4 \mathrm{ijk} \mathrm{lmn}$ & 62,1 ghijk \\
\hline IAC $80-508-3 \ldots \ldots \ldots \ldots \ldots$ & 26,3ghijkl & 66,0 fghijk \\
\hline IAC $-8 \ldots \ldots \ldots \ldots \ldots \ldots$ & $29,4 \mathrm{fghij}$ & 65,9 efghijk \\
\hline Cristalina ............... & 26,9ghijk & 65, efghijk \\
\hline IAC-11 $\ldots \ldots \ldots \ldots \ldots \ldots \ldots$ & 34,9 efg & 65,8 efghijk \\
\hline $\mathrm{UFV}-1 \ldots \ldots \ldots \ldots \ldots \ldots$ & $16,6 \mathrm{klmnop}$ & $70,4 \mathrm{defghij}$ \\
\hline IAC $78-2286 \ldots \ldots \ldots \ldots \ldots$ & 23,4hijklm & 70,1defghij \\
\hline IAC $79-1823 \ldots \ldots \ldots \ldots$ & 23,6 hijklm & 70,0defghi \\
\hline IAC Santa Maria $702 \ldots \ldots \ldots$ & $33,4 \mathrm{efgh}$ & $74,0 \mathrm{defgh}$ \\
\hline PI $229358 \ldots \ldots \ldots \ldots \ldots \ldots$ & 31,8efghi & 76,1 defg \\
\hline PI $171451 \ldots \ldots \ldots \ldots \ldots \ldots$ & 30,9 efghi & $78,3 \mathrm{cdef}$ \\
\hline IAC-10 $\ldots \ldots \ldots \ldots \ldots \ldots$ & 32,0efghi & $81,5 \mathrm{cde}$ \\
\hline IAC $-2 \ldots \ldots \ldots \ldots \ldots \ldots$ & 30,3 fghij & $82,4 \mathrm{~cd}$ \\
\hline D72-9601-1 ............. & $51,3 \mathrm{~cd}$ & $81,9 \mathrm{~cd}$ \\
\hline IAC $-12 \ldots \ldots \ldots \ldots \ldots$ & $56,4 b c$ & $88,9 \mathrm{bc}$ \\
\hline São Carlos . . . . . . . . . . . . & $42,1 \mathrm{de}$ & $93,8 \mathrm{ab}$ \\
\hline IAS $-5 \ldots \ldots \ldots \ldots \ldots \ldots$ & $64,3 \mathrm{~b}$ & $93,8 \mathrm{ab}$ \\
\hline Bossier $\ldots \ldots \ldots \ldots \ldots \ldots \ldots$ & 40,9 def & $94,8 \mathrm{ab}$ \\
\hline IAC Foscarim $31 \ldots \ldots \ldots \ldots$ & $55,9 \mathrm{bc}$ & $97,6 \mathrm{ab}$ \\
\hline Paraná ...................... & $92,6 \mathrm{a}$ & $99,4 \mathbf{a}$ \\
\hline$\ldots \ldots \ldots \ldots \ldots \ldots \ldots$ & 29,4 & 64,6 \\
\hline Coeficiente de variação . . . . . . . . . . & $13,5 \%$ & $11,5 \%$ \\
\hline
\end{tabular}

* Médias seguidas pela mesma letra náo diferem entre si pelo teste de Duncan a 5\%. 
QUADRO 5. Produção de grãos comerciais* de 35 germoplasmas de soja infestados por percevejos (média de quatro repetiçöes). Campinas

Tratamento

$\frac{\text { Produção** }}{1983 / 1984}$

$1984 / 1985$

$\mathrm{kg} / \mathrm{ha}$

PI $274453 \ldots \ldots \ldots \ldots \ldots \ldots \ldots \ldots$

IAC $80-598-2-4 \ldots \ldots \ldots \ldots \ldots \ldots$

Cristatina .................

IAC $-9 \ldots \ldots \ldots \ldots \ldots \ldots$

IAC $80-4228 \ldots \ldots \ldots \ldots \ldots \ldots \ldots$

PI $274454 \ldots \ldots \ldots \ldots \ldots \ldots \ldots \ldots$

IAC $73-228 \ldots \ldots \ldots \ldots \ldots$

IAC $80-508-3 \ldots \ldots \ldots \ldots \ldots \ldots$

IAC $78-2318 \ldots \ldots \ldots \ldots \ldots \ldots$

IAC $-7 \ldots \ldots \ldots \ldots \ldots \ldots$

IAC $80-596-2 \ldots \ldots \ldots \ldots \ldots \ldots$

IAC $-11 \ldots \ldots \ldots \ldots \ldots$

IAC $80-95-0-2 \ldots \ldots \ldots \ldots \ldots$

IAC $80-4213 \ldots \ldots \ldots \ldots \ldots \ldots$

IAC $79-1823 \ldots \ldots \ldots \ldots \ldots \ldots$

IAC $-10 \ldots \ldots \ldots \ldots \ldots \ldots$

PI $227687 \ldots \ldots \ldots \ldots \ldots \ldots \ldots \ldots$

UFV $-1 \ldots \ldots \ldots \ldots \ldots$

IAC $78-2286 \ldots \ldots \ldots \ldots \ldots \ldots$

IAC $80-55-1 \ldots \ldots \ldots \ldots \ldots$

IAC $80-612-5-2 \ldots \ldots \ldots \ldots \ldots \ldots$

IAC $80-572-2-1 \ldots \ldots \ldots \ldots \ldots \ldots \ldots$

Santa Rosa . . . . . . . . . . . . .

IAC Santa Maria $702 \ldots \ldots \ldots . . . .$.

IAC-8 . . . . . . . . . . .

D72-9601-1 .............

IAC $-2 \ldots \ldots \ldots \ldots \ldots \ldots \ldots$

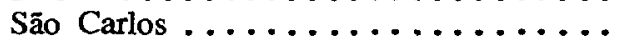

IAC $-12 \ldots \ldots \ldots \ldots \ldots$

IAS $-5 \ldots \ldots \ldots \ldots \ldots \ldots$

PI $229358 \ldots \ldots \ldots \ldots \ldots \ldots$

PI $171451 \ldots \ldots \ldots \ldots \ldots \ldots \ldots \ldots$

Bossier . . . . . . . . . . . . . .

IAC Foscarim $31 \ldots \ldots \ldots \ldots \ldots$

Paraná ..................
$3.675 \mathrm{ab}$

2.003 efgh

$2.804 \mathrm{cde}$

$3.527 \mathrm{abc}$

1.675fghij

$4.144 \mathrm{a}$

1.530 ghij

$1.920 \mathrm{fgh}$

1.639fghij

2.206defg

1.817 fghi

$3.038 \mathrm{bcd}$

2.322defg

1.667 fghij

1.984 efgh

$2.156 \mathrm{efgh}$

$2.253 \mathrm{defg}$

$2.519 \mathrm{def}$

1.647fghij

$2.783 \mathrm{cde}$

1.505ghij

2.009 efgh

$2.785 \mathrm{cde}$

1.851 fghi

1.668fghij

$1.521 \mathrm{ghij}$

$1.741 \mathrm{fghi}$

2.307defg

1.519ghij

$816 \mathrm{jk}$

1.546ghij

$1.895 \mathrm{fghi}$

1.278hij

1.025ijk

$402 k$ $\mathrm{kg} / \mathrm{ha}$

2.967a

$1.866 \mathrm{~b}$

$1.691 \mathrm{bc}$

$1.639 \mathrm{bcd}$

$1.639 \mathrm{bcd}$

$1.535 \mathrm{bcde}$

$1.419 \mathrm{bcdef}$

$1.286 \mathrm{bcdefg}$

1.285 bcdefg

$1.266 \mathrm{bcdefg}$

$1.256 \mathrm{cdefg}$

$1.235 \mathrm{cdefg}$

$1.224 \mathrm{cdefg}$

$1.195 \mathrm{cdefg}$

$1.173 \mathrm{cdefg}$

$1.152 \mathrm{cdefg}$

1.048defgh

1.048defgh

954efghi

905fghij

878fghijk

867fghijk

816fghijk

799fghijk

798 fghijk

726ghijk

666ghijkl

438hijklm

404ijklm

$334 \mathrm{jklm}$

$322 \mathrm{jklm}$

$280 \mathrm{klm}$

$1281 \mathrm{~m}$

851m

$31 \mathrm{~m}$

Média

2.036

1.010

Coeficiente de variação

$25,3 \%$

$36,1 \%$

* São os gräos sem nenhum defeito mais os grãos com sinal de ataque de percevejos, mas sem deformação.

** MÉdias seguidas pela mesma letra não diferem entre si pelo teste de Duncan a 5\%. 
QUADRO 6. Altura, produção, índice porcentual de dano de vagens (IPDV) e porcentagem de retenção foliar (PRF) de 35 genótipos de soja infestados por percevejos (médias de dois anos, quatro repetições cada ano). Campinas, 1983/1984 e 1984/1985

\begin{tabular}{|c|c|c|c|c|}
\hline Tratamento & Altura & Produção & IPDV & PRF \\
\hline & $\mathrm{cm}$ & $\mathrm{kg} / \mathrm{ha}$ & $\%$ & $\%$ \\
\hline PI $274453 \ldots \ldots \ldots \ldots \ldots \ldots \ldots$ & 169,5 & 3.321 & 11,1 & 18,7 \\
\hline PI $274454 \ldots \ldots \ldots \ldots \ldots \ldots \ldots$ & 177,5 & 2.839 & 12,1 & 12,5 \\
\hline IAC-9 $\ldots \ldots \ldots \ldots \ldots \ldots \ldots$ & 101,0 & 2.583 & 43,9 & 54,3 \\
\hline Cristalina ............... & 95,5 & 2.247 & 46,2 & 52,1 \\
\hline IAC-11 $\ldots \ldots \ldots \ldots \ldots \ldots \ldots$ & 98,0 & 2.136 & 50,3 & 15,1 \\
\hline IAC $80-598-2-4 \ldots \ldots \ldots \ldots \ldots$ & 60,0 & 1.934 & 27,0 & 5,2 \\
\hline IAC $80-55-1 \ldots \ldots \ldots \ldots \ldots$ & 64,5 & 1.844 & 42,2 & 15,1 \\
\hline UFV-1 $\ldots \ldots \ldots \ldots \ldots \ldots \ldots$ & 75,0 & 1.783 & 43,5 & 45,2 \\
\hline IAC $80-95-0-2 \ldots \ldots \ldots \ldots \ldots$ & 72,0 & 1.773 & 42,9 & 12,6 \\
\hline IAC-7 $\ldots \ldots \ldots \ldots \ldots \ldots$ & 89,5 & 1.736 & 40,3 & 32,4 \\
\hline Santa Rosa . . . . . . . . . . . . & 75,5 & 1.700 & 39,3 & 40,6 \\
\hline IAC $80-4228 \ldots \ldots \ldots \ldots \ldots \ldots$ & 101,5 & 1.657 & 26,3 & 7,1 \\
\hline IAC $-10 \ldots \ldots \ldots \ldots \ldots$ & 73,5 & 1.654 & 56,7 & 37,5 \\
\hline PI $227687 \ldots \ldots \ldots \ldots \ldots \ldots \ldots$ & 147,5 & 1.650 & 26,1 & 15,0 \\
\hline IAC $80-508-3 \ldots \ldots \ldots \ldots$ & 62,5 & 1.603 & 46,1 & 10,0 \\
\hline IAC $79-1823 \ldots \ldots \ldots \ldots$ & 85,5 & 1.578 & 46,8 & 38,3 \\
\hline IAC $80-596-2 \ldots \ldots \ldots \ldots$ & 57,5 & 1.536 & 34,7 & 10,6 \\
\hline IAC $73-228 \ldots \ldots \ldots \ldots \ldots$ & 64,0 & 1.474 & 30,6 & 4,7 \\
\hline IAC $78-2318 \ldots \ldots \ldots \ldots \ldots$ & 63,0 & 1.462 & 32,6 & 11,0 \\
\hline IAC $80-572-2-1 \ldots \ldots \ldots \ldots \ldots$ & 65,5 & 1.438 & 39,2 & 32,2 \\
\hline IAC $80-4213 \ldots \ldots \ldots \ldots \ldots$ & 67,0 & 1.431 & 31,6 & 31,5 \\
\hline São Carlos . . . . . . . . . . . & 67,5 & 1.372 & 67,9 & 57,5 \\
\hline IAC Santa Maria $702 \ldots \ldots \ldots$ & 118,5 & 1.325 & 53,7 & 61,0 \\
\hline IAC $78-2286 \ldots \ldots \ldots \ldots \ldots$ & 64,5 & 1.300 & 46,7 & 26,8 \\
\hline IAC-8 $\ldots \ldots \ldots \ldots \ldots \ldots$ & 85,5 & 1.233 & 47,6 & 48,1 \\
\hline IAC $-2 \ldots \ldots \ldots \ldots \ldots \ldots$ & 119,0 & 1.203 & 56,3 & 32,3 \\
\hline IAC $80-612-5-2 \ldots \ldots \ldots \ldots$ & 60,5 & 1.191 & 34,7 & 15,6 \\
\hline D72-9601-1 ............ & 48,0 & 1.123 & 66,6 & 49,2 \\
\hline PI $171451 \ldots \ldots \ldots \ldots \ldots \ldots$ & 39,0 & 1.087 & 54,6 & 14,3 \\
\hline IAC $-12 \ldots \ldots \ldots \ldots \ldots$ & 69,5 & 961 & 72,6 & 58,8 \\
\hline PI $229358 \ldots \ldots \ldots \ldots \ldots \ldots \ldots$ & 36,5 & 934 & 53,9 & 23,7 \\
\hline Bossier $\ldots \ldots \ldots \ldots \ldots \ldots \ldots$ & 60,0 & 703 & 67,8 & 61,1 \\
\hline IAS $-5 \ldots \ldots \ldots \ldots \ldots \ldots$ & 54,5 & 575 & 79,0 & 84,5 \\
\hline IAC Foscarim $31 \ldots \ldots \ldots$ & 86,0 & 555 & 76,7 & 61,2 \\
\hline Paraná ................ & 63,0 & 216 & 96,0 & 95,8 \\
\hline
\end{tabular}


A introdução PI 274453 é muito tardia (Quadro 1), e embora se tenha destacado pela produção e pouco dano sofrido, seu uso como fonte de resistência é duvidoso com base nesses resultados, porque foi a última a frutificar, e o baixo dano pode ser devido à evasão hospedeira.

Os coeficientes de correlação simples (r) entre a produção, índice porcentual de dano de vagem (IPDV) e porcentagem de retenção foliar (PRF) calculados acham-se no quadro 7. O dano provocado às vagens, estimado pelo IPDV, e a retenção foliar, pela PRF, são efeitos do ataque dos percevejos e estão correlacionados entre si. $O$ dano causado às vagens reduz a produção. As equaçōes de regressão linear entre essas variáveis foram as seguintes, respectivamente em 1983/84 e 1984/85:

$$
\begin{aligned}
& \text { Produção }(\mathrm{kg} / \mathrm{ha})=2822-26,7 \text { IPDV } \\
& \text { Produção }(\mathrm{kg} / \mathrm{ha})=2485-22,9 \text { IPDV. }
\end{aligned}
$$

A retençāo foliar também está relacionada com menor produção, embora esta relação seja menor que a anterior, entre o IPDV e a produção. As equações obtidas através de regressão linear entre a produção e a porcentagem de retenção foliar em 1983/1984 e 1984/1985 foram respectivamente:

$$
\begin{aligned}
& \text { Produção }(\mathrm{kg} / \mathrm{ha})=2192-15,4 \text { PRF } \\
& \text { Produção }(\mathrm{kg} / \mathrm{ha})=1592-10,1 \text { PRF. }
\end{aligned}
$$

QUADRO 7. Valores do coeficiente de correlação $r$, entre três variáveis, produção, porcen-

\begin{tabular}{|c|c|c|}
\hline \multirow{2}{*}{ Variáveis } & \multicolumn{2}{|c|}{ Valores de $\mathrm{r}$} \\
\hline & $1983 / 84$ & $1984 / 85$ \\
\hline $\operatorname{IPDV} \times \mathrm{PRF} \ldots \ldots \ldots \ldots \ldots$ & $0,81^{*}$ & $0,74 *$ \\
\hline Produção x IPDV $\ldots \ldots \ldots \ldots \ldots \ldots \ldots \ldots$ & $-0,60^{*}$ & $-0,84 *$ \\
\hline Produção $x$ PRF $\ldots \ldots \ldots \ldots \ldots \ldots \ldots \ldots$ & $-0,39 *$ & $-0,57^{*}$ \\
\hline
\end{tabular}
tagem de retenção foliar (PRF) e indice porcentual de dano de vagens (IPDV), obtidos com 35 germoplasmas de soja infestados de percevejos. Campinas, 1983/1984 e 1984/ $/ 1985$

* Valores significativos ao nível de $5 \%$ pelo teste $t$.

\section{SUMMARY}

\section{RESISTANCE OF SOYBEAN TO INSECTS. VI. PERFORMANCE OF VARIETIES IN RELATION TO STINK BUGS}

The performance of 35 soybean germplasms including 16 cultivars, 6 plant introductions and 13 lines derived from the soybean improve- 
ment program against stink bugs, was evaluated for two consecutive years, 1983/1984 and 1984/1985, in relation to the stink bugs (Hemiptera, Pentatomidae) species Piezodorus guildinii (Westwood), Euschistus heros (F.) and Nezara viridula (L.). The two experiments were conducted under field conditions at the Experimental Center of Instituto Agronômico located in Campinas, State of São Paulo, Brazil. A complete randomized block design with 35 treatments and 4 replications was used. The individual plot was a single row 2 meters long. Artificial infestations with adults were made to supplement the natural field infestation of stink bugs. The infestation level was moderate in the first year and severe in the second. Three criteria were used to judge the resistance to stink bugs: the percentual index of pod damage, the percentage of foliar retention after pod maturation and yield. The percentual index of pod damage is given by the formula $1 / 2$ ( $\%$ of pods with intermediate damage) $+\%$ of pods completely damaged. The percentage of foliar retention was visually estimated upon maturation of the pods. The cultivar Parana was the most susceptible treatment being recommended for use as a susceptible check for stink bugs. The lines IAC80-4228 and IAC80-598-2-4, obtained through the soybean breeding program for resistance to stink bugs, had good performance. They still present however some undesirable agronomic features and cannot be recommended for distribution to the farmers.

Index terms: soybean, Glycine $\max (\mathrm{L}$.$) Merrill, stink bugs, Pentatomidae, plant resis-$ tance, Piezodorus guildinï (Westwood), Nezara viridula (L.), Euschistus heros (F.).

\section{AGRADECIMENTOS}

Agradecimentos são devidos a Maria Oliveira de Barros, Archangelo Marion, Maria Inês Fonseca Jorge, Jaão Luiz da Silva e Aldo Fernandes, funcionários da Seção de Entomologia Fitotécnica, pelo auxílio na realização deste trabalho, e à Fundação de Amparo à Pesquisa do Estado de São Paulo (FAPESP), pelo auxílio concedido.

\section{REFERÊNCIAS BIBLIOGRÁFICAS}

LOURENÇÃO, A.L.; ROSSETTO, C.J. \& MIRANDA, M.A.C. de. Resistência de soja a insetos. III. Seleção de linhagens resistentes a percevejos. Bragantia, Campinas, 44(1): 77-86, 1985.

MIRANDA, M.A.C. de; ROSSETTO, C.J.; ROSSETTO, D.; BRAGA, N.R.; MASCARENHAS, H.A.A.; TEIXEIRA, J.P.F. \& MASSARIOL, A. Resistência de soja a Nezara viridula e Piezodorus guildinii em condiçôes de campo. Bragantia, Campinas, 38:181-188, 1979.

PANIZZI, M.C.C.; BAYS, I.A.; KIIHL, R.A.S. \& PORTO, M.P. Identificação de genotipos fontes de resistência a percevejos-pragas da soja. Pesquisa Agropecuária Brasileira, Brasília, 16(1):33-37, 1981. 
ROSSETTO, C.J.; LOURENÇÃO, A.L.; MIRANDA, M.A.C. de \& IGUE, T. Resistência de soja a insetos. II. Teste de livre escolha entre a linhagem IAC 73/228 e o cultivar Paraná, infestados por Nezara viridula (L.) em telado. Bragantia, Campinas, 43(1):141153, 1984.

; NAGAI, V.; IGUE, T.; ROSSETTO, D.\& MIRANDA, M.A.C. de. Preferência de alimentação de adultos de Diabrotica speciosa (Germar) e Cerotoma arcuata (Oliv.) em variedades de soja. Bragantia, Campinas, 40:179-183, 1981. 